\title{
Association of age at menarche with metabolic syndrome and its components in rural Bangladeshi women
}

Shamima Akter ${ }^{1,3}$, Subrina Jesmin ${ }^{1,2^{*}}$, Mazedul Islam ${ }^{1,3}$, Sayeeda Nusrat Sultana ${ }^{1,3}$, Osamu Okazaki ${ }^{3}$, Michiaki Hiroe ${ }^{3}$, Masao Moroi $i^{3}$ and Taro Mizutani ${ }^{2}$

\begin{abstract}
Background: Early age at menarche is associated with increased risk of metabolic syndrome in both China and the West. However, little is known about the impact of age at menarche and metabolic syndrome in South Asian women, including those from low-income country, where age at menarche is also falling. The aim of the present study was to investigate whether age at menarche is inversely associated with metabolic syndrome in Bangladeshi women, who are mostly poor and have limited access to and or poor health care facilities.

Methods: This community-based cross-sectional study was performed using 1423 women aged between 15-75 years from rural Bangladesh in 2009 and 2010. Metabolic syndrome was defined according to standard NCEP-ATP III criteria. Logistic regression was used to estimate the association between age at menarche and metabolic syndrome, with adjustment of potential confounding variables, including age, education, marital status, tobacco users, use of contraceptives and number of pregnancies.

Results: Early onset of menarche ( $<12$ years) as compared to late onset ( $>13$ years) was found to be associated with a higher prevalence of metabolic syndrome (odds ratio $=1.55 ; 95 \%$ confidence interval $=1.05-2.30$ ). Age at onset of menarche was also inversely associated with prevalence of high triglycerides $(P$ for trend $<0.01)$ and low high-density lipoprotein cholesterol $(P$ for trend $=0.01)$, but positively associated with prevalence of high fasting blood glucose ( $P$ for trend $=0.02$ ). However, no significant association was found between age at menarche, high blood pressure and elevated waist circumference.

Conclusion: Early onset of menarche might promote or trigger development of metabolic syndrome. Thus, knowledge of the history of age at onset of menarche may be critical in identifying women at risk of developing metabolic syndrome and those likely to benefit the most from early interventions.
\end{abstract}

Keywords: Age at menarche, Metabolic syndrome, Women, Rural Bangladesh

\section{Background}

Metabolic syndrome is a cluster of risk factors that include obesity, insulin resistance, dyslipidemia, and hypertension. Collectively, these factors increase the risk for cardiovascular disease and type 2 diabetes [1,2]. Over the past two decades, there has been a noticeable world-wide increase

\footnotetext{
* Correspondence: jsubrina@hdrcrp.org

${ }^{1}$ Health \& Disease Research Center for Rural Peoples (HDRCRP), 14/15, 1st Floor, Probal Housing Ltd., Shekertak (Adjacent to Shekertak Road 1), Mohammadpur, Dhaka 1207, Bangladesh

${ }^{2}$ Graduate School of Medicine, Faculty of Medicine, University of Tsukuba, Tsukuba, Ibaraki 305-8575, Japan

Full list of author information is available at the end of the article
}

in the prevalence of metabolic syndrome, including more recently in developing countries, where the prevalence has been much lower [3,4]. This new development has further compounded the challenges that the weak health system in developing countries is still struggling to cope with, i.e., communicable diseases [5]. The prevalence of metabolic syndrome in women tend to be greater than in men in many developing countries [4]. In view of these gender disparities, it is critical that we identify women at risk of contracting these diseases earlier on in life for effective interventions and outcomes.

\section{Biomed Central}

(c) 2012 Akter et al.; licensee BioMed Central Ltd. This is an Open Access article distributed under the terms of the Creative Commons Attribution License (http://creativecommons.org/licenses/by/2.0), which permits unrestricted use, distribution, and reproduction in any medium, provided the original work is properly cited. 
Menarche, the onset of menstruation in girls, indicates the attainment of full reproductive capacity. The age that menarche occurs varies and is dependent on the interaction between genetic and environmental factors and, in some cases, lifestyle factors [6,7]. The life style changes resulting from industrialization, decreased levels of physical activity and increased or excess intake of energy substrates has led to a rapid decrease in age at onset of menarche both in developed and more recently in developing countries [8-10]. Early onset of menarche has been shown to be associated with higher body mass index (BMI) [11-14], risk factor of cardiovascular diseases and its associated morbidity and mortality [15-17], as well as type 2 diabetes $[18,19]$. Therefore, in recent times, there has been increased interest in identifying whether an association exists between age at onset of menarche and metabolic syndrome, in order to better prevent and manage the myriad chronic non-communicable diseases that occur in women.

To date, few studies have reported the association between age at onset of menarche and metabolic syndrome $[16,20,21]$ and the bulk of the data from these studies were mainly obtained from the West [16,21], with very limited data from Asia [20]. Specifically, to our knowledge, no such study has been conducted so far on this issue in South Asian population or in low-income countries, including Bangladeshi women. The characteristics and conditions of South Asian women are substantially different from Western or Far East Asian populations. For instance, South Asian women tend to have a higher prevalence of abdominal obesity but lower BMI compared to Western and other Asian women [4]. Further, there are socioeconomic disparities between the different regions of the globe. The current mean age at menarche is 12.8 years in Bangladesh, which was considerably lower than the previous estimate [22]. Bangladesh is a low-income country with a GDP per capita income of 588 USD, an average life expectancy at birth of 67 years, and an adult literacy rate of about 55\% [23]. Besides, in Bangladesh more than onethird of the population lives below the poverty lines and are unable to even meet the barest of their basic needs [24], and women disproportionately constitute the bulk of the poor community. Thus, the goal of the present study was to clarify whether age at onset of menarche plays any etiological role in the development of metabolic syndrome in a low-income country by investigating existing of an association between age at onset of menarche and metabolic syndrome and its components. We hypothesized that age at onset of menarche is inversely associated with metabolic syndrome in rural Bangladeshi women.

\section{Methods}

\section{Study procedure}

The present study is a community-based cross-sectional study performed using women from rural Bangladesh between 2009 and 2010. A total of 1535 females aged $\geq 15$ years were selected using the stratified multistage random sampling. This sample size (1535) was sufficient to test all our formulated research hypotheses at the $5 \%$ level of significance, with a power of $80 \%(\beta=0.20)$. We used the World Health Organization's (WHO) STEPS approach (modified), which entails a stepwise collection of the risk factor data, based on standardized questionnaires covering demographic characteristics, somatic illnesses, somatic and mental symptoms, medications, life style, and health-related behavior (step 1), basic physical measures (step 2) and basic biochemical investigations, such as blood glucose and cholesterol (step 3). The women were recruited from 4 village communities located in Gabindagonj Upazilla (sub district) of Gaibandha district. The respondents were selected randomly after selecting the division, district, Upazila and villages, and were recruited through local announcements at community level and by house-to-house visits. The details of the study area have been described before in our previous study [25]. Gobindogonj Upazilla has a population of 514,696, with average household size of about 3.88, and the main occupation is agriculture, which is similar to other rural parts of Bangladesh [26]. The literacy rate of our target community is $45.8 \%$ and $39.5 \%$ for males and females, respectively, which is a bit lower than the average or overall rural literacy rate (54.39\% and $46.19 \%$ for male and female, respectively) in the country [26]. The data from participants were obtained through interviews and clinical examinations at mobile examination centers, where blood samples were also collected. The study was approved by the Ethical Committee of the Health and Disease Research Center of Rural Peoples (HDRCRP), Dhaka, Bangladesh, and conforms to the principles outlined in the Helsinki Declaration. Also all participating subjects gave their written informed consent before they were included in the study.

\section{Study subjects}

Out of 1535 women, the following subjects were excluded: those lacking information on triglyceride, high-density lipoprotein (HDL) cholesterol, and fasting blood glucose; missing information of age at onset of menarche, or missing information for any other covariates used in the main analysis. Participants with menarche later than 16 years were also excluded, because this is likely to be due to pathological status or recall error. Finally, a total of 1423 subjects remained in this study.

\section{Anthropometric and other variables}

Anthropometric measurements on individuals wearing light clothing and without shoes were conducted by well-trained examiners, as described here: height was 
measured to the nearest $0.1 \mathrm{~cm}$ using the portable stadiometer; weight was then measured in an upright position, to the nearest $0.1 \mathrm{~kg}$, using a calibrated balance beam scale; BMI was calculated as body weight $(\mathrm{kg})$ divided by the square of the body height $\left(\mathrm{m}^{2}\right)$; and waist circumference measurements were taken at the end of normal expiration, to the nearest $0.1 \mathrm{~cm}$, by measuring from the narrowest point between the lower borders of the rib cage and the iliac crest. Blood pressure was measured twice in the right arm in a sitting position using the standard mercury manometer and cuff, to the nearest $2 \mathrm{mmHg}$, with the initial reading taken at least 5 minutes after the subject was made comfortable, and again after an interval of 15 minutes. The average systolic and diastolic blood pressures were then estimated. Tobacco use, education, marital status, use of contraceptives, and experience of prior pregnancies were self-reported. Ever tobacco users were defined as those who are current tobacco users (smoked/chewed tobacco) and who had not smoked/chewed tobacco in the past 30 days preceding the survey, but have prior experience.

\section{Biochemical analysis}

Blood for biochemical analysis was obtained from the participants after a 10-12 hour overnight fast. The blood sample was collected using the standard blood sample collection procedure. Immediately after collection of blood and labeling of the blood vials, the samples were transported to the National Centre for Global Health and Medicine (NCGM), Japan, for biochemical assessment. For analysis, the serum was immediately separated from the blood by centrifugation for evaluating plasma concentration of lipids. Triglycerides levels were measured by lipoprotein lipase method (Wako Chemicals, Tokyo, Japan), HDL cholesterol was measured with the Determiner-L kit (Kyowa Co Ltd, Tokyo, Japan). Fasting plasma glucose levels were measured with the Hexokinase G-6-PDH kit (Wako Pure Chemical Industries Ltd, Osaka, Japan).

\section{Assessment of age at menarche}

Age at onset of menarche was defined as age at the first menstrual bleeding, assessed in full years. This information was obtained by a personal interview at the time of survey. The question was open ended "at what age did you have your first menstrual period (menarche)?" For the present study, analysis of age at onset of menarche was categorized into three categories $(<12$ years, $12-13$ years, and $>13$ years). The reliability of the questionnaire on age at onset of menarche was confirmed in our follow up study. The intra-class correlation coefficient was 0.89 for continuous variables of age at onset of menarche.

\section{Definition of metabolic syndrome and risk factors}

Metabolic syndrome and metabolic risk factors were defined according to standard criteria of the National Cholesterol Education Program's Adults Treatment Panel III (NCEP-ATP III) [27]. Three or more of the following components were defined as having metabolic syndrome, namely: a) abdominal obesity, as measured by a waist circumference of $\geq 88 \mathrm{~cm}$; b) high fasting blood glucose $(\geq 110 \mathrm{mg} / \mathrm{dL}$ or $\geq 6.1 \mathrm{mmol} / \mathrm{L})$ or patients diagnosed with diabetes; c) high triglycerides ( $\geq 150 \mathrm{mg} / \mathrm{dL}$ or $\geq 1.7 \mathrm{mmol} / \mathrm{L}$ ); d) low HDL cholesterol $(<50 \mathrm{mg} / \mathrm{dL}$ or $<1.29 \mathrm{mmol} / \mathrm{L})$; e) high blood pressure $(\geq 130 / \geq 85 \mathrm{mmHg}$ ) or subjects diagnosed with hypertension. Also, participants who at the time of the study reported to be on anti-hypertensive or anti-diabetic medications (insulin or oral agents) were considered as having high blood pressure or high fasting blood glucose, respectively.

\section{Statistical analysis}

The characteristics of the participants of the study were presented according to increasing categories of age at onset of menarche and trend associations were assessed using linear regression analysis for continuous variables or Mantel-Haenszel chi-square test for categorical variables, with ordinal numbers 1-3 assigned to increasing categories of age at menarche.

To evaluate the magnitude of the association between age at onset of menarche and metabolic syndrome and its components (obesity, high triglycerides, low HDL cholesterol, high blood pressure, and high fasting blood glucose), we estimated adjusted odds ratio (OR) and 95\% confidence interval with multivariable logistic regression models. Two models were used considering highest age at onset of menarche category as reference category. The first models were adjusted for age (year, continuous), and education (illiterate, have formal education). The second models were further adjusted for marital status (currently married or others), tobacco users (ever or never), use of contraceptives (ever or never), and number of pregnancies (continuous). Trend association was assessed by assigning ordinal numbers 1-3 assigned to increasing categories of age at menarche. Two-sided $P$ values $<0.05$ were regarded as statistically significant. All analyses were performed using statistical software STATA version 12.0 (Lakeway Drive, College Station, Texas USA).

\section{Results}

Table 1 shows the characteristics of the study subjects, based on the categories of age at onset of menarche. Age at onset of menarche was positively associated with levels of fasting blood glucose and HDL cholesterol but was inversely associated with levels of triglyceride. Subjects with higher age at menarche were more likely to be 
currently married and have no formal education but less likely to be tobacco users. Prevalence of metabolic syndrome across categories of age at onset of menarche was also presented (Table 1). As age at onset of menarche increased, prevalence of metabolic syndrome was found to decrease, although it was not found to be statistically significant $(P$ for trend $=0.16)$.

Table 2 shows odds ratio of metabolic syndrome and its components, according to increasing categories of age at onset of menarche. Age at onset of menarche was inversely associated with prevalence of metabolic syndrome in age and education adjusted model $(P$ for trend $=$ 0.02). Additional adjustment for other covariates, including marital status, tobacco and contraceptive usage, and number of pregnancies, were found to somewhat attenuates the association but still found to be statistically significant $(P$ for trend $=0.03$ ). Subjects with the lowest age at onset of menarche had 1.55 times higher odds of having metabolic syndrome than those with late onset of menarche. Age at onset of menarche was also inversely associated with prevalence of high triglyceride $(P$ for trend $<0.01$ ) and low HDL cholesterol ( $P$ for trend $=0.01$ ) but was found to be positively associated with high fasting blood glucose $(P$ for trend $=0.02$ ). High blood pressure and elevated waist circumference was not significantly associated with age at onset of menarche $(P$ for trend $=$ 0.83 and 0.23 , respectively).

\section{Discussion}

In the present cross-sectional study, we use rural Bangladeshi women to examine the relationship between age at onset of menarche and metabolic syndrome. We found that age at onset of menarche was inversely associated with metabolic syndrome in these women after controlling for potential confounding variables. Age at onset of menarche was also inversely associated with some components of metabolic syndrome, including high triglyceride, and low HDL cholesterol, but was found to be positively associated with high fasting blood glucose. To our knowledge this is the first study to reveal an association between age at onset of menarche and metabolic syndrome in South Asian women and in a low-income country.

The inverse association between age at onset of menarche and metabolic syndrome are consistent with most of the previous studies from both the Western [16,21] and Far Eastern Asian populations [20]. The present findings are also in agreement with two previous studies of same line conducted in the US [28] and China [29]. Therefore, the present data reconfirms these earlier findings and demonstrate their prevalence in Bangladeshi women, who are mostly poor, have lower BMI but higher waist circumference and have lower mean age at menarche compared with their Western and Far Eastern Asian counterparts [4,22], suggesting that early onset of menarche may increase prevalence of metabolic syndrome, irrespective of race and ethnicity.

Age at onset of menarche was inversely associated with high levels of plasma triglyceride, and low HDL cholesterol components in our study. The significant inverse association between age at onset of menarche and high triglycerides in our study are concordant with those

Table 1 Characteristics of study population by age at menarche

\begin{tabular}{|c|c|c|c|c|}
\hline \multirow[t]{2}{*}{ Characteristic } & \multicolumn{4}{|c|}{ Age at menarche (years) } \\
\hline & $<12$ & $12-13$ & $>13$ & Trend $\mathrm{P}^{\mathbf{b}}$ \\
\hline $\bar{n}$ & 488 & 583 & 352 & \\
\hline Current age (years) & $40.91 \pm 11.68^{a}$ & $42.12 \pm 12.92$ & $42.72 \pm 13.57$ & 0.04 \\
\hline $\mathrm{BMI}\left(\mathrm{kg} / \mathrm{m}^{2}\right)$ & $21.43 \pm 4.94$ & $21.96 \pm 4.01$ & $22.26 \pm 4.05$ & 0.08 \\
\hline Number of pregnancies & $3.50 \pm 2.13$ & $3.44 \pm 2.08$ & $3.36 \pm 2.03$ & 0.35 \\
\hline Currently married (\%) & 80.94 & 88.16 & 94.32 & $<0.01$ \\
\hline Ever use of contraceptives (yes, \%) & 19.67 & 17.50 & 19.03 & 0.74 \\
\hline Use of tobacco products (ever, \%) & 31.56 & 19.04 & 7.10 & $<0.01$ \\
\hline Education (illiterate, \%) & 52.05 & 54.37 & 59.66 & 0.03 \\
\hline Waist circumference (cm) & $77.20 \pm 9.32$ & $76.96 \pm 8.86$ & $76.38 \pm 7.90$ & 0.19 \\
\hline Fasting blood glucose (mmol/L) & $5.81 \pm 2.12$ & $6.29 \pm 2.99$ & $6.78 \pm 4.65$ & $<0.01$ \\
\hline Triglyceride (mg/dL) & $131.5 \pm 102.8$ & $130.5 \pm 105.4$ & $127.9 \pm 119.2$ & 0.03 \\
\hline HDL cholesterol (mg/dL) & $36.54 \pm 18.7$ & $40.89 \pm 31.05$ & $48.71 \pm 42.40$ & $<0.01$ \\
\hline Systolic blood pressure (mmHg) & $115.46 \pm 20.26$ & $116.73 \pm 21.74$ & $116.70 \pm 19.46$ & 0.28 \\
\hline Diastolic blood pressure (mmHg) & $74.53 \pm 10.00$ & $75.74 \pm 10.34$ & $75.29 \pm 10.53$ & 0.23 \\
\hline Metabolic syndrome (\%) & 26.14 & 25.73 & 22.13 & 0.16 \\
\hline
\end{tabular}

Abbreviation: BMI, body mass index; HDL, high-density lipoprotein.

${ }^{a}$ Values are mean $\pm S D$, all such values. 
Table 2 Association of age at menarche with metabolic syndrome and its components

\begin{tabular}{|c|c|c|c|c|}
\hline & \multicolumn{4}{|c|}{ Age at menarche (years) } \\
\hline & $<12$ & $12-13$ & $>13$ & Trend $\mathrm{P}^{\mathrm{a}}$ \\
\hline \multicolumn{5}{|c|}{ Metabolic syndrome ${ }^{b}$} \\
\hline Model 1 & $1.60(1.09-2.35)$ & $1.38(0.97-1.97)$ & 1.00 (reference) & 0.02 \\
\hline Model 2 & $1.55(1.05-2.30)$ & $1.36(0.95-1.94)$ & 1.00 (reference) & 0.03 \\
\hline \multicolumn{5}{|c|}{ Central obesity } \\
\hline Model 1 & $1.67(0.92-3.05)$ & $1.82(1.02-3.27)$ & 1.00 (reference) & 0.11 \\
\hline Model 2 & $1.53(0.82-2.85)$ & $1.73(0.96-3.11)$ & 1.00 (reference) & 0.23 \\
\hline \multicolumn{5}{|c|}{ High fasting blood glucose } \\
\hline Model 1 & $0.59(0.42-0.83)$ & $0.73(0.54-0.99)$ & 1.00 (reference) & $<0.01$ \\
\hline Model 2 & $0.65(0.46-0.93)$ & $0.76(0.56-1.04)$ & 1.00 (reference) & 0.02 \\
\hline \multicolumn{5}{|c|}{ High triglyceride } \\
\hline Model 1 & $2.27(1.59-3.25)$ & $1.82(1.31-2.53)$ & 1.00 (reference) & $<0.01$ \\
\hline Model 2 & $1.97(1.36-2.84)$ & $1.70(1.22-2.35)$ & 1.00 (reference) & $<0.01$ \\
\hline \multicolumn{5}{|c|}{ Low HDL cholesterol } \\
\hline Model 1 & $1.99(1.32-2.98)$ & $1.41(1.00-2.00)$ & 1.00 (reference) & $<0.01$ \\
\hline Model 2 & $1.71(1.12-2.60)$ & $1.30(0.91-1.84)$ & 1.00 (reference) & 0.01 \\
\hline \multicolumn{5}{|c|}{ High blood pressure } \\
\hline Model 1 & $0.94(0.66-1.35)$ & $1.01(0.73-1.43)$ & 1.00 (reference) & 0.71 \\
\hline Model 2 & $1.05(0.72-1.52)$ & $1.08(0.77-1.53)$ & 1.00 (reference) & 0.83 \\
\hline
\end{tabular}

Model 1 adjusted for age (year, continuous), education (illiterate, have formal education).

Model 2 adjusted for age (year, continuous), education (illiterate, have formal education), marital status (currently married or others), use of tobacco products (ever or never), ever use of contraceptives (yes or no), and number of pregnancies (continuous).

a Based on multiple logistic regression analysis, with ordinal numbers 1-3 assigned to increasing categories of age at menarche.

${ }^{\mathrm{b}}$ Metabolic syndrome is defined as presence of at least 3 of the following criteria.

Obesity component (Waist circumference $\geq 88 \mathrm{~cm})$, high fasting blood glucose $(\geq 110 \mathrm{mg} / \mathrm{dL}$ ), high triglycerides ( $\geq 150 \mathrm{mg} / \mathrm{dL})$, low-high-density lipoprotein (HDL) cholesterol ( $<50 \mathrm{mg} / \mathrm{dL}$ ), high blood pressure; systolic blood pressure (SBP $\geq 130 \mathrm{~mm} \mathrm{Hg}$ ) or diastolic blood pressure (DBP $\geq 85 \mathrm{~mm} \mathrm{Hg}$ ).

of the previous studies [20,21,29], conducted so far. Data are sparse and conflicting as to whether early onset of menarche is inversely associated with increasing prevalence of low HDL cholesterol. The inverse association between age at onset of menarche and low HDL cholesterol revealed in our study is consistent with a previous Chinese study [29]. However, the majority of previous studies found no significant association between age at onset of menarche and prevalence of low HDL cholesterol [20,21]. Although currently there is no clear evidence that early onset of menarche increases the prevalence of low HDL cholesterol, based on the present findings, we could expect to see significant benefits in late onset of menarche in as far as metabolic syndrome prevalence is concerned, as well as lower prevalence of HDL cholesterol. Such trends and associations also provides us with some important insights that may play a crucial role in the development of metabolic syndrome preventive interventions in these rural communities where prevalence of low HDL cholesterol is significantly high [30].

Unlike the expected association between age at onset of menarche and other metabolic components, age at onset of menarche was not inversely associated rather positively associated with fasting blood glucose. These results have not been reported previously so far by earlier studies. Contrarily to the findings of the present studies, most of the previous studies either found no significant association $[16,29,31]$ or inverse association between age at onset of menarche and high fasting blood glucose $[21,28,32]$. In addition, in a previous Chinese study, a threshold effect of early menarche ( $<12.5$ years) on elevated fasting blood glucose was found [20]. The results of our current study contract these earlier findings. At this time, there is no apparent reason to account for this association. However, it is possible that there may be some other factors responsible for this association other than age at onset of menarche alone, which may include various aspects of lifestyle of the study subjects.

The observed association between late onset of menarche and high fasting blood glucose could be attributed, in part, to the current unhealthy lifestyle and low quality diet in women with late onset of menarche. Alternatively, these present findings may reflect differences between the age groups and circumstances surrounding 
their generations since the subjects with late menarche were relatively older in our study population. The relatively late onset of menarche among older women are likely linked to various malnutrition factors caused either by inflammation or famine, caused by flooding or following Bangladesh's war for independence in 1971 [33]. However, after 1976, the economy began to steadily advance. Based on stratified analysis, according to the mean current age of women (41.48 years), a clear positive association between age at onset of menarche and high fasting blood glucose was found only among older women (data not shown). However, no significant association was found among the younger women (data not shown). Therefore, collectively, these trends suggest that the lifestyle and life circumstances of the older women may be responsible for the positive association between age at onset of menarche and high fasting blood glucose, which are different from the experiences of the younger women.

The mechanism underlying the positive association between age at onset of menarche and metabolic syndrome for now remains unclear. It has been suggested that childhood obesity may influence the age at onset of sexual maturation and hence the age at the onset of menarche $[34,35]$. In addition, it was found that obesity in childhood is associated with metabolic syndrome in adolescence and adulthood [16,36]. Therefore, it is possible that the observed association between early onset of menarche and metabolic syndrome may be explained by the link between childhood obesity and metabolic syndrome. Lastly, differences in the pattern and levels of sex hormone differences over the lifespan of the women may account for the observed association between early menarche and metabolic syndrome. This speculation warrants a prospective study for clarity.

The major strengths of the present study include the fact that it is based on: a) a community-wide survey drawn from the general population, b) the anthropometric data are derived from actual measurements rather than self-reported, c) and are adjusted for potential confounding variables. However, despite these strengths, the present study has some limitations that are worth mentioning: First, since the ages at onset of menarche were self-reported, there could have been some error in reporting. However, the extent of such an error should have been minimal since onset of menarche is a discrete physiological event and a key milestone in the lives of girls, thus continues to be memorable event even in adulthood. This conclusion is consistent with data from previous studies that showed that the actual age at onset of menarche and recall of age at menarche was not found to differ even after 33 years later $[11,37]$. The second limitation is that it was not possible to adjust for pubertal BMI due to a lack of information of the self- reported $\mathrm{BMI}$ at the onset of menarche or menstruation. This is because in a low-income country like Bangladesh, rural women and girls are not always aware about their BMI, so it is difficult to assess the accuracy of selfreported pubertal BMI. Thirdly, although we adjusted for important confounders, the possibility of residual confounding cannot be completely ruled out. Finally, since our study is a cross-sectional it could have selection bias during case recruitment because only rural women from lower socio-economic class were used. Thus, these results cannot be generalized to the whole community of Bangladeshi women nation-wide. However, because there are minimal differences in rural communities, the present results may relevant to the larger nation-wide community of Bangladeshi women in rural areas.

In conclusion, the present study shows that early onset of menarche is associated with metabolic syndrome in rural Bangladeshi women. Knowledge of the history of age at onset of menarche may be essential and help in identifying women at risk or those likely to have metabolic syndrome later in life. Early identification of women at risk may help to prevent metabolic syndrome, which is also the precursor of type 2 diabetes and cardiovascular diseases.

\section{Abbreviations}

BMI: Body mass index; HDL cholesterol: High-density lipoprotein cholesterol.

\section{Competing interests}

The authors declare that they have no competing interests.

\section{Authors' contributions}

The authors responsibilities are as follows- SA: conducted data analysis, drafted the manuscript and had primary responsibility for final content; SJ: conducted data collection, arranged financial support, extensively reviewed and edited the manuscript; and all authors: were involved in interpretation of results and revision of the manuscript and approved the final version of the manuscript.

\section{Acknowledgement}

The authors thank the study subjects for their cooperation and participation. This work was supported in part by a Grant-in-Aid for Scientific Research (overseas academic) from the Ministry of Education, Culture, Sports, Science and Technology of Japan (22406025, 23406037, 23406016, 23406029), and Japan Society for the promotion of Science. Research grant from Uehara Memorial Foundation, Japan and World Diabetes Foundation (WDF),

Denmark have also supported a part of this work.

\section{Author details}

${ }^{1}$ Health \& Disease Research Center for Rural Peoples (HDRCRP), 14/15, 1st Floor, Probal Housing Ltd., Shekertak (Adjacent to Shekertak Road 1), Mohammadpur, Dhaka 1207, Bangladesh. ${ }^{2}$ Graduate School of Medicine, Faculty of Medicine, University of Tsukuba, Tsukuba, Ibaraki 305-8575, Japan. ${ }^{3}$ National Center for Global Health and Medicine (NCGM), 1-21-1 Toyama, Shinjuku-ku, Tokyo 162-8655, Japan.

Received: 16 August 2012 Accepted: 4 November 2012

Published: 9 November 2012

\section{References}

1. Ford ES, Li C, Sattar N: Metabolic syndrome and incident diabetes: current state of the evidence. Diabetes Care 2008, 31(9):1898-1904.

2. Mottillo S, Filion KB, Genest J, Joseph L, Pilote L, Poirier P, Rinfret S, Schiffrin EL, Eisenberg MJ: The metabolic syndrome and cardiovascular risk a systematic 
review and meta-analysis. J Am Coll Cardiol 2010,

56(14):1113-1132.

3. Cameron AJ, Shaw JE, Zimmet PZ: The metabolic syndrome: prevalence in worldwide populations. Endocrinol Metab Clin North Am 2004, 33(2):351-375

4. Misra A, Khurana L: Obesity and the metabolic syndrome in developing countries. J Clin Endocrinol Metab 2008, 93(11 Suppl 1):S9-S30.

5. Xu K, Evans DB, Kawabata K, Zeramdini R, Klavus J, Murray CJ: Household catastrophic health expenditure: a multicountry analysis. Lancet 2003, 362(9378):111-117.

6. Elks CE, Perry JRB, Sulem P, Chasman DI, Franceschini N, He C, Lunetta KL, Visser JA, Byrne EM, Cousminer DL: Thirty new loci for age at menarche identified by a meta-analysis of genome-wide association studies. Nat Genet 2010, 42(12):1077-1085.

7. Karapanou O, Papadimitriou A: Determinants of menarche. Reprod Biol Endocrinol 2010, 8:115.

8. Graham MJ, Larsen U, Xu X: Secular trend in age at menarche in China: a case study of two rural counties in Anhui Province. J Biosoc Sci 1999, 31(2):257-267.

9. Moisan J, Meyer F, Gingras S: A nested case-control study of the correlates of early menarche. Am J Epidemiol 1990, 132(5):953-961.

10. Wattigney WA, Srinivasan SR, Chen W, Greenlund KJ, Berenson GS: Secular trend of earlier onset of menarche with increasing obesity in black and white girls: the Bogalusa Heart Study. Ethn Dis 1999, 9(2):181-189.

11. Must A, Phillips S, Naumova E, Blum M, Harris S, Dawson-Hughes B, Rand W: Recall of early menstrual history and menarcheal body size: After 30 years, how well do women remember? Am J Epidemiol 2002, 155(7):672-679.

12. Pierce $M B$, Leon $D A$ : Age at menarche and adult BMI in the Aberdeen children of the 1950s cohort study. Am J Clin Nutr 2005, 82(4):733-739.

13. Remsberg KE, Demerath EW, Schubert CM, Chumlea WC, Sun SS, Siervogel RM: Early menarche and the development of cardiovascular disease risk factors in adolescent girls: the Fels Longitudinal Study. J Clin Endocrinol Metab 2005, 90(5):2718-2724

14. van Lenthe FJ, Kemper CG, van Mechelen W: Rapid maturation in adolescence results in greater obesity in adulthood: the Amsterdam Growth and Health Study. Am J Clin Nutr 1996, 64(1):18-24.

15. Jacobsen B, Oda K, Knutsen S, Fraser G: Age at menarche, total mortality and mortality from ischaemic heart disease and stroke: the Adventist Health Study, 1976-88. Int J Epidemiol 2009, 38(1):245-252

16. Kivimäki M, Lawlor DA, Smith GD, Elovainio M, Jokela M, KeltikangasJärvinen $L$, Vahtera J, Taittonen $L$, Juonala M, Viikari JSA: Association of age at menarche with cardiovascular risk factors, vascular structure, and function in adulthood: the Cardiovascular Risk in Young Finns study. Am J Clin Nutr 2008, 87(6):1876-1882.

17. Lakshman R, Forouhi NG, Sharp SJ, Luben R, Bingham SA, Khaw KT, Wareham NJ, Ong KK: Early age at menarche associated with cardiovascular disease and mortality. J Clin Endocrinol Metab 2009, 94(12):4953-4960

18. He C, Zhang C, Hunter DJ, Hankinson SE, Louis GMB, Hediger ML, Hu FB: Age at menarche and risk of type 2 diabetes: results from 2 large prospective cohort studies. Am J Epidemiol 2010, 171(3):334-344.

19. Lakshman R, Forouhi N, Luben R, Bingham S, Khaw K, Wareham N, Ong K: Association between age at menarche and risk of diabetes in adults: results from the EPIC-Norfolk cohort study. Diabetologia 2008, 51(5):781-786

20. Heys M, Schooling CM, Jiang C, Cowling BJ, Lao X, Zhang W, Cheng KK, Adab P, Thomas GN, Lam TH, et al: Age of menarche and the metabolic syndrome in China. Epidemiology 2007, 18(6):740-746.

21. Stöckl D, Meisinger C, Peters A, Thorand B, Huth C, Heier M, Rathmann W, Kowall B, Stöckl H, Döring A: Age at menarche and its association with the metabolic syndrome and its components: results from the KORA F4 Study. PLoS One 2011, 6(10):e26076

22. Rah JH, Shamim AA, Arju UT, Labrique AB, Rashid M, Christian P: Age of onset, nutritional determinants, and seasonal variations in menarche in rural Bangladesh. J Health Popul Nutr 2009, 27(6):802-807.

23. World Bank: World Development Indicator. USA: World Bank; 2012. http:// data.worldbank.org/country/bangladesh

24. The world fact book. http://www.cia.gov/library/publications/the-worldfactbook/fields/2046.htm
25. Jesmin S, Islam R, Shahidul Islam SM, Sultana SN, Zaedi S, Yamaguchi N, Iwashima $Y$, Hiroe $M$, Watanabe $T$ : Comprehensive assessment of metabolic syndrome among Rural Bangladeshi Women. BMC Publ Health 2012, 12(1):49.

26. Bangladesh Bureau of Statistics (BBS): Population and Housing Census 2011. Dhaka, Bangladesh: Ministry of planning; 2012

27. Grundy SM, Cleeman JI, Daniels SR, Donato KA, Eckel RH, Franklin BA, Gordon DJ, Krauss RM, Savage PJ, Smith SC Jr, et al: Diagnosis and management of the metabolic syndrome: an American Heart Association/National Heart, Lung, and Blood Institute Scientific Statement. Circulation 2005, 112(17):2735-2752.

28. Frontini MG, Srinivasan SR, Berenson GS: Longitudinal changes in risk variables underlying metabolic Syndrome $X$ from childhood to young adulthood in female subjects with a history of early menarche: the Bogalusa Heart Study. Int J Obes Relat Metab Disord 2003, 27(11):1398-1404.

29. Feng Y, Hong X, Wilker E, Li Z, Zhang W, Jin D, Liu X, Zang T, Xu X, Xu X: Effects of age at menarche, reproductive years, and menopause on metabolic risk factors for cardiovascular diseases. Atherosclerosis 2008, 196(2):590-597.

30. Jesmin S, Mia MS, Islam A, Islam MR, Sultana SN, Zaedi S, Yamaguchi N, Okazaki O, Moroi M, Kimura S: Prevalence of metabolic syndrome among rural Bangladeshi women. Diabetes Res Clin Practice 2012, 95(1):e7-e9.

31. Chen L, Zhang C, Yeung E, Ye A, Mumford SL, Wactawski-Wende J, Schisterman EF: Age at menarche and metabolic markers for type 2 diabetes in premenopausal women: The BioCycle Study. J Clin Endocrinol Metab 2011, 96(6):E1007-E1012.

32. Stöckl D, Döring A, Peters A, Thorand B, Heier M, Huth C, Stöckl H, Rathmann W, Kowall B, Meisinger C: Age at menarche is associated with prediabetes and diabetes in women (aged 32-81 years) from the general population: the KORA F4 Study. Diabetologia 2012, 55(3):681-688.

33. Chowdhury AK, Huffman SL, Curlin GT: Malnutrition, menarche, and marriage in rural Bangladesh. Soc Biol 1977, 24(4):316-325.

34. Dunger DB, Lynn Ahmed M, Ong KK: Effects of obesity on growth and puberty. Best Pract Res Clin Endocrinol Metab 2005, 19(3):375-390.

35. Freedman DS, Khan LK, Serdula MK, Dietz WH, Srinivasan SR, Berenson GS: The relation of menarcheal age to obesity in childhood and adulthood: the Bogalusa heart study. BMC Pediatr 2003, 3:3.

36. Biro FM, Wien M: Childhood obesity and adult morbidities. Am J Clin Nutr 2010, 91(5):1499S-1505S

37. Bosetti C, Tavani A, Negri E, Trichopoulos D, La Vecchia C: Reliability of data on medical conditions, menstrual and reproductive history provided by hospital controls. J Clin Epidemiol 2001, 54(9):902-906.

doi:10.1186/1743-7075-9-99

Cite this article as: Akter et al:: Association of age at menarche with metabolic syndrome and its components in rural Bangladeshi women. Nutrition \& Metabolism 2012 9:99.

\section{Submit your next manuscript to BioMed Central and take full advantage of:}

- Convenient online submission

- Thorough peer review

- No space constraints or color figure charges

- Immediate publication on acceptance

- Inclusion in PubMed, CAS, Scopus and Google Scholar

- Research which is freely available for redistribution 